\title{
Philosophiques
}

\section{Du contrat à la communication : Habermas critique Rawls}

\section{Bjarne Melkevik}

Volume 24, numéro 1, printemps 1997

Avez-vous lu Rawls?

URI : https://id.erudit.org/iderudit/027424ar

DOI : https://doi.org/10.7202/027424ar

Aller au sommaire du numéro

\section{Éditeur(s)}

Société de philosophie du Québec

\section{ISSN}

0316-2923 (imprimé)

1492-1391 (numérique)

Découvrir la revue

Citer cet article

Melkevik, B. (1997). Du contrat à la communication : Habermas critique Rawls. Philosophiques, 24(1), 59-70. https://doi.org/10.7202/027424ar

\section{Résumé de l'article}

Dans ce texte, l'auteur analyse et réfléchit sur la critique posée par le philosophe allemand Jürgen Habermas à l'encontre du libéralisme politique de son homologue américain John Rawls. Insistant sur quelques thèmes clés, l'auteur se penche, dans un premier temps, sur la critique formulée à l'encontre du modèle d'autonomie politique et individuelle développé par Rawls. Dans un deuxième temps, il examine la position habermasienne à l'égard du discours deformation des normes élaboré par Rawls. Cette étude fait ressortir certaines lacunes de la théorie rawlsienne au regard du monde contemporain et propose une approche alternative tenant davantage compte de Vintersubjectivité et de la démocratie. 


\title{
DU CONIRAT À LA COMMUNICATION : HABERMAS CRIIIQUE RAWLS
}

\author{
PAI \\ Bjarne Melkevik
}

\begin{abstract}
RÉSUMÉ : Dans ce texte, l'auteur analyse et réfléchit sur la critique posée par le philosophe allemand Jürgen Habermas à l'encontre du libéralisme politique de son homologue américain John Rawls. Insistant sur quelques thèmes clés, l'auteur se penche, dans un premier temps, sur la critique formulée à l'encontre du modèle d'autonomie politique et individuelle développé par Rawls. Dans un deuxième temps, il examine la position habermasienne à l'égard du discours de formation des normes élaboré par Rawls. Cette étude fait ressortir certaines lacunes de la théorie rawlsienne au regard du monde contemporain et propose une approche alternative tenant davantage compte de l'intersubjectivité et de la démocratie.

ABSTRACT: In this text, the author analyses and considers the critic of German philosopher Jürgen Habermas over the political liberalism of his american homologue John Rawls. The author first looks at the critics formulated against Rawls's model of political and individual autonomy. The author then proceeds to examine Habermas's position regarding Rawls's discourse on norm formation. This study shows certain lacunas in Rawls's theory in regard of today's society and proposes an alternative approach which would take into account democracy and intersubjectivity.
\end{abstract}

Les philosophies de John Rawls et de Jürgen Habermas ont profondément influencé la philosophie contemporaine au cours des trois dernières décennies. John Rawls, par sa Théorie de la justice parue en 1971 , a réintroduit au cœur de la préoccupation contemporaine la question d'une société et des institutions justes ${ }^{1}$. Jürgen Habermas a èlaboré, pour sa part, avec sa Théorie de l'agir communicationnel, une philosophie originale qui, au confluent de différentes préoccupations, s'efforce de repenser le sens de la modernité ${ }^{2}$. Si nous avons longtemps observé une complémentarité entre ces deux projets, et mème une utilisation pragmatique de Rawls par Habermas, tout vient de basculer avec le projet, chez J. Rawls, d'un "libéralisme politique "qui consacre la distance réelle existant entre ces deux

1. John Rawls, Théorie de la justice, Paris, Seuil, 1987.

2. Jürgen Habermas, Théorie de l'agir communicationnel, Paris, Fayard, 1987. 
philosophies ${ }^{3}$. En fait, depuis le début des années 1980 , et surtout à partir de 1985, Rawls abandonne progressivement sa théorie morale initiale de mème que sa théorie de la raison pratique ${ }^{4}$. Il relativise l'héritage des Lumières, décrivant le procéduralisme de cette période comme " sectaire ", il ouvre la porte au "communautarisme", il affaiblit tout projet d'universalisme argumenté et il se réfugie dans un conservatisme visant d'abord la stabilité. Dans cette optique, la critique que Habermas lui adresse ne mesure pas uniquement la distance qui sépare leurs philosophies respectives, elle revêt, en outre, une valeur symbolique, celle de rompre l'enchantement que Rawls a longtemps exercé sur la philosophie juridique et politique contemporaine.

L'objectif de notre article consiste à analyser et à réfléchir sur la critique de Habermas à l'endroit de Rawls ${ }^{5}$. Nous verrons comment Habermas désapprouve le libéralisme politique de Rawls, et comment il en démontre les apories théoriques. Selon Habermas, le projet de libéralisme politique de Rawls est une théorie sans prise sur les enjeux primordiaux de notre modernité. Cette critique nous intéresse dans la mesure où elle permet de jeter un regard inédit sur le passage d'une philosophie du "contrat "vers une philosophie de la communication. Par cette approche, il va sans dire que nous choisissons le modèle d'une philosophie communicationnelle au détriment de la philosophie de Rawls ${ }^{6}$. Nous croyons, en effet, qu'une philosophie communicationnelle a, par son souci pour le monde concret des acteurs et des enjeux sociaux et politiques réels, un avantage non négligeable sur la philosophie hypothético-logique de Rawls. Mais ne brülons pas les étapes encore à franchir.

Dans notre ètude, nous insisterons sur quelques thèmes clefs se rapportant à la philosophie du droit. Nous voulons d'abord, dans un premier temps, examiner la critique habermasienne du modèle d'autonomie politique et individuelle chez Rawls pour ensuite examiner comment Habermas critique le discours de formation des normes élaboré par Rawls. Dans ces analyses, c'est le contraste explicatif qui constitue la trame de la réflexion.

3. J. Rawls, Libéralisme politique, Paris, P.U.F.. 1995. Voir aussi du mème auteur, Justice et Démocratie, Paris, Seuil, 1993.

4. Voir Chandran Kukathas \& Philip Pettit, Rawls. A Theory of Justice and its Critics, Stanford, Stanford University Press, 1990.

5. J. Habermas, - Reconciliation through the Public Use of Reason : Remarks on John Rawls's Political Liberalism " dans The Journal of Philosophy. vol. 92, $n^{\circ} 3$, mars 1995, p. 109-131. Notre analyse repose principalement sur cet article, mais aussi sur la critique de Rawls dans Between Facts and Norms. Contributions to a Discourse Theory of Law and Democracy. Cambrigde (Mass.), MIT Press, 1996; " Human Rights and Popular Sovereignty : The Liberal and Republican Versions * dans Ratio Juris, vol. 7. $n^{\circ} 1$, mars 1994, p. 1-13; "Three Normative Models of Democracy " dans Constellations, vol. $1, \mathrm{n}^{\circ} 1,1994, \mathrm{p} .1-10$.

6. Voir J. Rawls, "Reply to Habermas ", dans The Joumal of Philosophy, vol. 92, $n^{\circ} 3$, mars 1995, p. 132-180. Observons que Rawls, dans sa réplique, ne développe pas de nouveaux arguments, mais essaie d'invalider la philosophie de Habermas comme "doctrine comprëhensive ". 


\section{Critique du modèle rawlsien de l'autonomie politique et individuelle}

Il convient d'abord de dire quelques mots à propos de l'objectif recherché par Habermas dans sa critique de Rawls sur ce thème. En fait, Habermas présente le problème de l'hèritage de la modernité en ce qui concerne l'autonomie (individuelle et politique) comme une lutte entre une conception liberale et une conception républicaine? ${ }^{7}$. La conception libérale a privilégié des droits fondamentaux libéraux, les libertés des modernes, en faisant de ces droits le fondement de l'État. Les républicains, par contre, ont privilégié les droits politiques de participation, les libertés des Anciens, en insistant sur la pratique de lautodétermination des citoyens. Dans la perspective de Habermas, les philosophes les plus perspicaces, tels Kant et Rousseau, ont tenté d'éviter un surinvestissement de l'un ou l'autre de ces deux versants de la modernité au profit d'un arbitrage raisonnable entre les deux. Aux yeux de Habermas, Rawls poursuit ce travail d'arbitrage, mais en s'appuyant sur un fondement philosophique déficient, à savoir une philosophie de la conscience dont l'insuffisance sera aggravée par son nouveau projet de libéralisme politique. L'enjeu de la critique du modèle rawlsien par Habermas n'est donc pas seulement de corriger un quelconque parti-pris philosophique, mais, plus profondément, d'inscrire l'intersubjectivité communicationnelle contre le soliloque de la conscience libérale. Nous le verrons d'abord au sujet de l'autonomie individuelle, ensuite en ce qui a trait à l'autonomie politique, autrement dit à la conception rawlsienne de la souveraineté, et, enfin, synthétiquement, au sujet de l'enjeu sous-jacent du sens démocratique.

\section{L'autonom ie ind ividuel le}

L'objection que Habermas adresse à la conception rawlsienne de l'autonomie individuelle est double; d'un côté, il la critique en tant que monologue et, d'un autre côté, il désapprouve la conception étriquée de l'individu introduite par Rawls. Précisons qu'il ne s'agit ici, chez Rawls comme chez Habermas, que du sens de l'autonomie individuelle dans son horizon juridique, et nullement métaphysique.

Examinons d'abord la conception qu'a Rawls de l'autonomie individuelle. Rawls considère que la source d'un système de droits et de libertés se réfère à l'idêe d'une société conçue comme un système juste de coopération. Les représentants des citoyens ont pour tâche de se mettre d'accord sur les termes d'une collaboration soumise à des conditions raisonnables. Comme participants à une telle coopération, les citoyens ont préalablement, comme Rawls le dit, deux facultés morales leur permettant de contribuer à cette entreprise ${ }^{\theta}$. D'abord, la capacité de se référer à un sens de la justice ; ensuite, la capacité de choisir une conception du bien. L'une se référe au

7. J. Habermas, "Reconciliation through the Public Use of Reason : Remarks on John Rawls's Political Liberalism *, p. 127. Voir aussi " Three Normative Models of Democracy " dans Constellations, vol. $1, \mathbf{n}^{0} 1,1994$, p. $1-10$; "Human Rights and Popular Souvereignty", dans Ratio Juris, vol. 7, $\mathbf{n}^{\circ} \mathbf{1}$, 1994. p. 1-13.

8. J. Rawls, Libéralisme politique, p. 43, 114 ss. 
"raisonnable ", l'autre au "rationnel ". Le sens de cette vision de l'autonomie individuelle est, comme l'affirme Rawls, que le libéralisme politique espère satisfaire la demande libérale traditionnelle de justifier le monde social d'une façon acceptable * selon le tribunal de compréhension propre à chaque individu ${ }^{10}$ ".

Cette façon d'envisager l'autonomie individuelle relève foncièrement d'un monologue, selon Habermas. L'autonomie individuelle est thématisée dans la perspective du " je "seulement. C'est la logique du modèle rawlsien qui transforme ultimement la multitude des " je " en " nous " politique. Une telle conception, d'après Habermas, demeure dépendante des prémisses d'une philosophie de la conscience, c'està-dire qu'elle est rivée au modèle d'un sujet solitaire. Ce modèle n'a pas besoin de l'argumentation entre les citoyens. Il n'a besoin que de modèles logico-politiques qui affirment juridiquement et politiquement les individus comme étant autonomes.

En fait, Habermas critique une conception de l'autonomie personnelle qui ne considère l'agent que sous l'angle d'un modèle hypothétique. Le modèle de Rawls ignore systématiquement la compréhension que l'agent raisonnable peut atteindre collectivement. Ce modèle méconnaît délibérément la compréhension réflexive que chaque individu peut acquẽrir dans l'espace public.

\section{L'autonomie politique ou la souverain eté}

La critique qu'il adresse à Rawls s'êclaire en abordant la question de l'autonomie politique. L'enjeu est pour Habermas la question du sens démocratique et comment celui-ci devient déficitaire dans le système rawlsien au bénéfice d'un faisceau de "principes " non thématisable. Car si Habermas comme Rawls partagent l'idée de la souveraineté du peuple comme base de la politique, elle n'a pas le méme sens pour chacun d'eux. Chez Rawls, la souveraineté du peuple se résume, selon Habermas, en une logique du choix rationnel institutionnellement prédéterminé et biaisé. Cette logique met largement les institutions à l'abri des enjeux politiques et démocratiques.

Nous pouvons aborder le thème de lautonomie politique rawlsienne en insistant sur la critique que Habermas fait de la métaphore de la position originelle. Rawls utilise cette image pour introduire le modèle de l'autonomie politique désirable ${ }^{11}$. En fait, Rawls pense que la position originelle peut ètre un moyen démocratique pour assurer et conduire à un accord juste. Sa théorie de la représentation suppose qu'une personne, au fait de la rivalité des doctrines compréhensives et dans un souci de neutralité à leur égard, a besoin d'une méthode de médiation politique "neutre "ou, comme Rawls le dit maintenant, "indépendante " (freestanding) ${ }^{12}$ pour fonder les normes et institutions adéquates. Certes, toute personne peut ultimement utiliser cette méthode, mais Rawls affirme qu'il s'agit

9. Ibid., p. 76 ss.

10. J. Rawls, "Reply to Habermas " dans The Journal of Philosophy. vol. 92. $\mathrm{n}^{\circ} 3$, mars 1995 , p. 146 , note 28 . Rawls se réfère et endosse l'opinion de Jeremy Waldron, Liberal Rights, New York, Cambridge, 1993, p. 61.

11. J. Rawls, Libéralisme politique, p. 47 ss.

12. Ibid., p 35. 
d'une mèthode qui, d'une façon privilégiée, peut être utilisée par des personnes ayant un "mandat "politique et juridique général de la part de la société ${ }^{13}$. Il s'agit ainsi d'une méthode qui pourrait être utilisée par les instances juridiques et politiques pour comprendre, interpréter et instaurer des politiques adéquates. Rawls suppose donc que le fait que toute personne raisonnable puisse vérifier, et à l'occasion contester, l'utilisation de cette méthode, assure une assise démocratique à ces instances juridiques et politiques.

La critique de Habermas consiste à dire que cette théorie met les institutions à l'abri d'une réflexion démocratique élargie. Pourquoi des individus entièrement autonomes requerraient-ils une théorie de la représentation qui ne les pose pas comme entièrement autonomes ? Pour Habermas, les individus n'ont pas besoin d'une telle représentation aussi longtemps que les citoyens sont conçus comme des personnes morales qui disposent d'un sens de la justice et de la capacité de se référer à leur propre conception du bien. Si tel est le cas, comme le prétend Habermas, Rawls ne fait que déplacer les caractéristiques d'une personne morale, sous les contraintes d'un modèle spécifique et abstrait de la rationalité, vers ce même modèle. Il introduit une pensée circulaire de l'auto-justification qui ne sert qu'à fournir des " atouts "aux juristes constitutionnels, dans la tradition juridique des pays occidentaux, en les instituant comme ultimes "fondements " et ultimes " juges".

Contre cette façon de concevoir l'autonomie politique, Habermas introduit l'idéal selon lequel " les citoyens sont autonomes politiquement uniquement s'ils peuvent se voir eux-mèmes réciproquement comme auteurs des lois auxquelles ils sont soumis comme destinataires individuels ${ }^{14}$ ". Il constate que ce n'est pas la vision de Rawls. Habermas poursuit sa critique des positions de Rawls et soutient que si ce ne sont que les citoyens qui peuvent rationnellement être désignés comme fondement et base, l'autonomie politique doit ètre entière et ne peut être propulsée dans un modèle hypothètico-logique présupposé apte à prendre rationnellement en charge lautonomie politique des citoyens. C'est le sens de la modernité juridique et politique qui est ainsi en jeu pour Habermas. Il considère que chez Rawls, l'homme moderne est " désapproprié " en tant qu'intersubjectivement responsable de la construction de la vie en commun. En fait, Habermas identifie le projet de la modernité comme "la nécessité de puiser en soi-mème tout ce qui relève des normes ${ }^{15}$ ". Dès lors, l'homme a besoin de toutes ses ressources, de toutes ses facultés, de toutes ses expériences. L'introduction d'un modèle hypothéticologique comme celui de Rawls et la hiérarchie de valeurs et de normes qu'il implique deviennent incompatibles avec ce projet. Lhomme ne puise pas en lui-mème chez Rawls, il puise dans le modèle hypothétique du philosophe.

13. Ibid., p. 280 ss et Justice et démocratie, p. 189.

14. J. Habermas, "Reconciliation through the Public Use of Reason : Remarks on John Rawls's Political Liberalism, p 130. Notre traduction : "Citizens are politically autonomous only if they can view themselves jointly as authors of the laws to which they are subject as individual addressees..

15. J. Habermas, Le discours philosophique de la modernité, Paris, Gallimard, 1988 , p. 23. 
En fait, cette critique met à nu ce que la théorie de Rawls n'est pas : lautonomie politique ne fait pas référence à la prise de parole dans l'espace public. Il n'implique pas un espace public permettant aux citoyens de "se voir eux-mèmes, réciproquement ", comme auteurs et destinataires des lois.

\section{Le sens démocratique}

La critique habermasienne de l'autonomie individuelle et politique chez Rawls peut se résumer à la question du rôle de la démocratie à l'égard des institutions. En fait, si l'autonomie, individuelle et politique, ne peut avoir de sens que dans une communauté politique, la conception de la démocratie que véhicule la théorie de Rawls est. selon Habermas, déficitaire.

Habermas n'a que peu de sympathie pour cette conception de la démocratie. Il la juge non acceptable politiquement et non pertinente empiriquement. Le modèle proposé par Rawls ne peut susciter l'assentiment effectif des citoyens et il ne peut ètre renouvelé pour maintenir une culture démocratique. Aux yeux de Habermas, ce modèle froid n'appelle pas à l'engagement démocratique. C'est un modèle qui ne suscite pas de participation.

Rawls introduit, selon Habermas, une conception limitée de la démocratie. Il la critique comme une conception dont le rôle principal serait de mettre les institutions démocratiques à l'abri des enjeux politiques et démocratiques effectivement existants. En fait, la position originelle n'est qu'une construction logico-formelle introduisant, selon Habermas, des restrictions non justifiées démocratiquement. Il s'agit d'une construction qui introduit des réserves dont la définition relève de la compétence du philosophe, ou plus probablement du juriste libéral constitutionnel. C'est alors à eux d'établir les limites à l'intêrieur desquelles s'exercent les intèrêts privés et politiques. Ces restrictions, tout comme l'enjeu démocratique, sont hors de la portée des citoyens. Finalement, Rawls ne fait que poursuivre la tendance inaugurée par J. Stuart Mill qui confine la direction politique et juridique à une "classe politique " $\operatorname{compétente~}^{16}$; une "classe politique " éclairée dont le rōle ultime est la stabilité " sociale " et la " tolérance " idéologique.

Dans sa critique de Rawls, Habermas propose sa propre conception de la démocratie. D'après lui, la démocratie se définit par la participation totale et entière de tous les citoyens ${ }^{17}$. La démocratie, c'est l'espace public assurant un échange de plus en plus élargi d'arguments et de raisons qui peuvent à la fois éclairer les individus et servir le processus de formation d'une volonté commune, communicationnellement partagée. La démocratie doit faire en sorte que les individus se définissent par leurs prises de parole. Celles-ci mobilisent leurs expériences, font appel au réservoir symbolique des

16. J. Stuart Mill, L'utilitarisme, Paris, Flammarion, 1988, p. 51-53.

17. B. Melkevik, "Habermas et l'État de droit. Le modèle communicationnel du droit et la reconstruction réflexive de l'État de droit contemporaine " dans Ayoub, Melkevik et Robert, éditeurs, L'Amour des bis. La crise de la loi modeme dans les sociétés démocratiques, Québec, P.U.L. et Paris, L'Harmattan, 1996. 
mondes vécus et utilisent les différentes formes de rationalités de la modernité. En somme, selon Habermas, la démocratie repose sur un droit de participation entier et non biaisè.

N'est-il pas significatif, en ce sens, d'observer que si Habermas a construit son opposition à Rawls selon le schème de la libertè des Modernes versus la liberté des Anciens, en soulignant la différence comme étant celle des droits moraux et des droits de participation, Rawls pour sa part définit ce mème schème comme une différence entre droits moraux et droit à l'égalité, ou encore comme " des valeurs de la liberté et de l'égalité ${ }^{18}$ ". Rawls introduit ainsi un contresens historique et philosophique, car les Anciens ne se préoccupaient guère de l'égalité, mais bien des droits de participation démocratiques; ce contresens philosophique nous met cependant sur la piste de l'aporie constitutive du projet rawlsien d'un libéralisme politique. Celui-ci ne peut thematiser l'essence d'un droit de participation entièrement démocratique qu'au risque de voir s'écrouler un échafaudage philosophique soigneusement construit.

\section{Critique du discours rawlsien de la fondation des normes}

La critique que Habermas adresse à Rawls sur la question de la fondation des normes permet d'observer le contraste entre ces deux discours rivaux de fondation de normes en problematisant le contractualisme de Rawls au profit d'une fondation communicationnelle des normes. Habermas structure sa critique selon trois axes ${ }^{19}:$ l) les parties peuvent-elles comprendre les intérêts suprêmes dautrui uniquement sur la base d'un égoïsme rationnel dans le concept de la position originelle ? 2) Les droits fondamentaux peuvent-ils etre assimilés aux biens premiers ? 3) Le voile d'ignorance peut-il garantir l'impartialité du jugement? Dans la présentation qui suit, nous respecterons cet ordre et ajouterons en 4) une synthèse et quelques réflexions.

\section{La position or iginelle}

Sans surprise, Habermas adresse le reproche suivant à Rawls : la position originelle ne met en cuvre qu'un égoïsme rationnel ${ }^{20}$. Abordons cet énoncé en approfondissant la critique habermasienne à l'égard de la rationalité de la poursuite des fins, ou stratégique, inhérente au modèle rawlsien.

La position originelle de Rawls est en fait une métaphore, une image, qui se veut analogue aux théories du contrat social moderne ${ }^{21}$. Ainsi, Rawls postule que le juste peut ètre défini intellectuellement, et voulu politiquement, au terme d'un calcul d'intérêts avantages/coūts

18. J. Rawls, Libéralisme politique, p. 29.

19. J. Habermas, "Reconciliation through the Public Use of Reason : Remarks on John Kawls's Political Liberalism ", p. 112.

20. J. Habermas, "Reconciliation through the Public Use of Reason : Remarks on John Rawls's Political Liberalism", p. 111-113. Voir aussi Morale et communication, p. 87-89, 100, 131 et 137 ; De l'éthique de la discussion, Paris, Cerf, 1992.

21. J. Rawls, Libéralisme politique. Pour une critique, voir : Jean Hampton, "Contracts and Choices : Does Rawls Have a Social Contract Theory ? " dans The Joumal of Philosophy, vol. 77, 1980, p. 315-338. 
qui, envisagé subjectivement, ne vise que le "bon "ou le "juste " pour le moi. La position originelle fait appel à la conception rawlsienne de l'autonomie individuelle que nous avons analysée auparavant: c'est le "je "qui choisit. Les individus, sous un voile d'ignorance, ne cherchent même pas, à proprement parler, à se mettre à la place d'autrui. Certes, ils peuvent imaginer toutes sortes de situations sociales et politiques possibles, mais il s'agit de positions qu'ils n'occupent pas et qu'ils ne connaissent pas non plus.

La position originelle fonde un égoïsme rationnel aux yeux de Habermas en excluant un moment "moral "véritable au sens de la philosophie pratique, à savoir " fonder " les normes comme étant partagées et validèes intersubjectivement. Jean-Marc Ferry observe avec justesse que la position rawlsienne met plutôt en ouvre un décentrement " paradoxal " de telle manière qu'une " considération égoïste suffirait à motiver : je peux ètre l'autre ". Elle met en œuvre une logique où le " je "doit se mettre à la place d'autrui. Aux yeux de Habermas, le voile d'ignorance sert ainsi à introduire un calcul égoïste sans tenir compte des enjeux concrets. En fait, ce calcul égoïste utilise l'hypothèse des conséquences pour le moi, sans pour autant thématiser empiriquement ces conséquences. Pour Habermas, le résultat consiste en un calcul égocentrique ne pouvant pas rendre compte de ce qui est également juste pour tous.

Comment les adhérents à la position originelle de Rawls peuventils, rétorque Habermas, "comprendre le fait que des citoyens autonomes respectent les intérēts des autres sur la base de principes justes et non uniquement pour des raisons d'intérêts propres, qu'ils peuvent avoir une obligation de loyauté, qu'ils souhaitent être convaincus de la légitimité des arrangements existant et d'une politique appropriée par un usage public de leur raison, etc. "Ils ne le peuvent pas, selon Habermas. Ils ne le pourront qu'en développant le "point de vue moral "d'une façon qui puisse satisfaire les intèrêts de tous et qui pourra recueillir l'assentiment de toutes les personnes impliquées dans une discussion réelle. Mais une telle position est loin de celle de Rawls.

\section{Les droits fondamentaux}

La deuxième critique que Habermas adresse au discours de la fondation des normes chez Rawls concerne sa conception des droits fondamentaux ${ }^{24}$ : les droits fondamentaux peuvent-ils ètre assimilés aux "biens premiers ", comme le soutient Rawls? Le droit est-il un "bien"?

Rawls identifie, en effet, les "droits fondamentaux "à un bien premier dans le sens précis que c'est un bien dont les citoyens ont besoin en tant que personnes égales et libres. Dans la liste qu'il dresse des biens premiers, les droits fondamentaux sont mis en

22. Jean-Marc Ferry, Philosophie de la communication 2. Justice politique et démocratie procédurale. Paris, Cerf, 1994, p. 54.

23. J. Habermas, "Reconciliation through the Public Use of Reason : Remarks on John Rawls's Political Liberalism ". p. 112.

24. Ibid., p. 113-116. 
exergue $^{25}$. Le fond de la pensèe rawlsienne exprime que les biens premiers reposent sur une base publique de comparaisons objectives permettant de rendre compte des conditions sociales des citoyens : le fait d'être libre. Ainsi, le terme "bien " se réfère à une conception de ce qu'est, selon nous, une vie humaine méritant d'être vécue, et les droits sont des "biens " parce qu'ils nous assurent et procurent cette possibilité de vie humaine ${ }^{26}$. Il s'agit d'une conception où les droits ne se partagent pas intersubjectivement, mais appartiennent à chaque individu sous le mode de l'avoir.

La critique de Habermas consiste à dire que les droits fondamentaux sont chez Rawls réduits à une perspective instrumentale. C'est une condition nécessaire pour que les personnes puissent réaliser des plans pour la vie qu'elles estiment, à tort ou à raison, ètre adéquate. Habermas considère que cette conception des droits fondamentaux est une variante d'une représentation qui peut ètre associée à la fois à l'aristotélisme et à l'utilitarisme ${ }^{27}$. Regrettons que Habermas n'approfondisse pas cette association subtile de Rawls avec l'utilitarisme; il fait cependant remarquer que le fond du problème est bien que Rawls associe le processus de fondation des normes à un processus téléologique, structuré selon la logique d'une personne et de son action. Autrement dit, c'est la valeur "utilitaire" des droits qui justifie leur existence.

Habermas oppose le sens moderne du droit à cette conception : un droit issu du savoir moral et pratique d'individus ayant fait du droit l'horizon de leur vie commune. Pourquoi Rawls veut-il soustraire les droits fondamentaux à ce savoir moral et pratique? Pourquoi veut-il fonder les droits fondamentaux de façon individualiste, alors que leur sens même concerne le vivre-ensemble? Il ne s'agit pas d'un quelconque conflit entre droit subjectif et droit collectif, mais plus précisement du rôle de l'autonomie politique dans l'élaboration des droits. Le processus restreint utilisé par Rawls dans la construction de l'autonomie individuelle et politique conduit à biaiser la médiation entre la conception libérale des droits fondamentaux et la conception républicaine. Son modèle impose que les droits fondamentaux s'écrivent sur le mode de biens individuel, là ou les enjeux sont intersubjectifs. La première catégorie de droits fondamentaux qui sera exclue est d'ailleurs le droit de participation politique, qui ne peut pas s'écrire sur ce mode.

Dans la conception des "droits-biens " chez Rawls, Habermas ne voit que le signe du refus d'admettre le rôle de "l'usage public de la raison "en ce qui concerne les droits fondamentaux. Rawls se construit un abri sécuritaire contre les discussions, les raisonnements, les argumentations qui peuvent se tenir dans l'espace public au moment de se donner des droits fondamentaux.

25. J. Rawls, Libéralisme politique, p. 224.

26. Voir William Rehg, Insight and Solidarity. The Discourse Ethics of Jürgen Habermas, Berkeley, University of Califomia Press, 1994, p. 124-125 ; voir aussi p. 125-134.

27. J. Habermas, "Reconciliation through the Public Use of Reason : Remarks on John Rawls's Political Liberalism ", p. 114. 


\section{Le voile d'ignorance}

La troisième critique que Habermas adresse à Rawls, ainsi qu'à son discours de fondation des normes valides conteste la thèse voulant que le voile d'ignorance puisse garantir l'impartialité du jugement ${ }^{28}$. Chez Rawls, le voile d'ignorance donne une pareille garantie en imposant d' oublier " qui nous sommes, d'où nous venons et ce que nous avons vécu. Le voile d'ignorance que nous posons devant nos yeux pour rendre opérationnel le processus de fondation de normes sert, comme le dit Rawls, à une réflexion impartiale ${ }^{29}$. La position originelle représente dans le système rawlsien une idée régulatrice par laquelle s'opère l'accord de toutes les considérations bien pesées.

La notion d'impartialité est, pour Habermas, liée à l'idée d'information des participants ${ }^{30}$. Si les participants n'ont pas accès à toute l'information possible les concernant et concernant la société, comment pouvons-nous, rétorque Habermas, prétendre que leur jugement est "impartial "? Un jugement non éclairé peut-il être dit "impartial "? Habermas ne voit ici qu'une sorte d'aveuglement volontaire, qu'une restriction des informations recevables et que l'introduction d'une surdétermination idéologique programmée à l'avance. En fait, Rawls a introduit une base normative qui de l'extérieur, c'est l'expression de Habermas ${ }^{31}$, doit arbitrer le flux d'informations qui vient de la société profondément divisée de notre modernité. Le voile d'ignorance agit comme un filtre qui empéche la prise en compte des expériences moralement signifiantes et des différentes formes d'apprentissage. Rawls étouffe en effet, selon Habermas, le flux d'informations sociales, politiques et culturelles, de telle sorte que le voile d'ignorance doit harmoniser uniquement les informations recevables selon les principes initiaux.

L'alternative, pour Habermas, n'est pas de se fermer à des expériences moralement significatives de notre modernité, mais de s'ouvrir pour une prise en compte. Sur le plan empirique, Habermas n'a aucun problème à montrer comment notre modernité juridique a effectivement et honteusement pu se fermer à de telles expériences. Le mouvement ouvrier a historiquement fait les frais d'une telle fermeture. L'expérience morale des ouvriers n'était simplement pas jugée pertinente pour le droit du $X I X^{e}$ siècle. Aujourd'hui, c'est le féminisme qui tente de thématiser l'expérience morale des femmes. Devons-nous nous fermer, comme nous le suggère Rawls, à ces expériences et rejeter l'information qu'elles peuvent nous apporter? Dans l'affirmative, pouvons-nous croire que ces mouvements, et d'autres encore, voudront accorder leur confiance à notre modernité juridique ? Celui que l'on n'écoute pas, n'est-il pas déjà traité comme un ennemi ? L'insistance d'Habermas quant au rōle primordial de l'information réfère par conséquent à l'usage public de la raison. L'usage

28. Ibid., p. 116-119.

29. J. Rawls, Libéralisme politique, p. 8, 78 et 82 .

30. J. Habermas, "Reconciliation through the Public Use of Reason : Remarks on John Rawls's Political Liberalism ", p. 116.

31. Ibid., p. 119. 
public de la raison inhérent à la perspective habermassienne requiert un flux d'informations, contrairement à la conception rawlsienne.

Contre Rawls, Habermas introduit par conséquent une façon tout à fait différente de considérer l'impartialité. Il la définit comme suit : "Seul est impartial le point de vue à partir duquel sont universalisables les normes mêmes qui, parce qu'elles incarnent manifestement un intérét commun à toutes les personnes concernées, peuvent escompter une adhésion générale et gagner, dans cette mesure, une reconnaissance intersubjective ${ }^{32}$ ". Il s'agit d'une conception de l'impartialité qui, contrairement à celle de Rawls, accepte et requiert toute information, toute expérience moralement pertinente.

\section{Quelques réflexions}

La critique de Habermas ayant trait au discours de fondation des normes sociales de Rawls soulève la question de la signification du "point de vue moral ".

La critique que nous venons d'analyser souligne que Rawls n'arrive pas à respecter les exigences d'un " point de vue moral ". Rawls se trouve, selon Habermas, dans une impasse, car il thématise cette exigence à partir d'un point de vue unique, celui du " je n, comme c'est le cas pour l'impératif catégorique de Kant. Il ne suffit pas pour Habermas que chaque individu procède, de façon privée, à une délibération cherchant à justifier les normes fondamentales. Ce qui est nécessaire, c'est que les individus concernés approuvent et reconnaissent intersubjectivement la validité des normes proposées. Habermas veut ainsi situer le "point de vue moral "sur l'axe de l'intersubjectivité. Il ne suffit plus de savoir qu'une personne est prête à se soumettre à la logique hypothétique de Rawls, il faut se demander plus concrètement ce que tous veulent ensemble. Habermas thématise "un point de vue moral "dont l'intersubjectivité fait référence à l'horizon d'une "volonté commune " communicativement partagée. Il est ainsi significatif que la question de validité des normes sociales ne puisse plus être le résultat d'une production logico-hypothétique interne comme chez Rawls. Chez Habermas, la production des normes relève des forces dêmocratiques et leur validation sera soumise à l'appréciation de tous.

\section{Bilan et questions}

Concluons notre article en dressant un bilan et en introduisant quelques pistes de réflexion.

Le sens de la critique habermasienne est à l'effet que la philosophie de Rawls a ses racines au XIX siècle. Le projet de Rawls n'est qu'une version modernisée de la tradition de la philosophie de conscience. C'est une philosophie qui se perpétue comme si le tournant linguistique n'avait jamais eu lieu. Ce qui revient constamment, c'est l'absence d'une intersubjectivité réelle et démocratique chez Rawls. Le sens du projet rawlsien risque de se réduire à une profession de foi dans les institutions politiques et juridiques des pays libres, pluralistes et démocratiques. Il s'agit en fait d'une

32. J. Habermas. Morale et comununication, p. 86. 
profession de foi dans le sens précis que son discours de fondation des normes débouche sur le "tribunal des principes", à savoir la Constitution et son interpretation, que seules les Cours suprêmes sont en pratique autorisées à interpréter.

L'absence d'un dialogue ouvert et démocratique, aussi bien pour fonder les institutions qu'en ce qui concerne l'interprétation et le schème de compréhension, hypothéquera le projet de Rawls. Suivant Habermas, Rawls a construit une "âme philosophique "et tenté d'animer le projet " constitutionnaliste "moderne. Mais cette " âme philosophique " ne peut concrètement être gérée que par une institution qui est supposée formée par le choix politique des hommes et des femmes vertueuses. Et encore. comme l'indique Habermas : il s'agit d'une " âme "qui nous échappe, qui se substitue à nous et qui, à la manière de l'hèritage des Lumières, travaille comme Raison raisonnante.

Le projet de Rawls représente-t-il une théorie dont nous avons besoin ? Force est de constater que tout ce que dit Habermas prouve le contraire, qu'il constitue la doctrine d'une retraite sécuritaire, sinon l'abdication du pouvoir entre les mains d'une "classe politique "prétendument éclairée et compétente. L'alternative n'est-elle donc pas de radicaliser notre compréhension de ce qu'exige la démocratie? Sinon, de faire le pari d'une conception radicale de la démocratie, comme le fait Habermas. Selon nous, il faut tenir ce pari.

Faculté de droit

Université Laval 